\title{
VOCABULAIRE DE L'IRRITATION \\ DANS LE DISCOURS DU MAROC ORIENTAL (CAS DE JERADA ET AIN BÉNI MATHAR)
}

\author{
Mohammed MALKI*
}

BIBLID [1133-8571] 26 (2019) 20.1-19.

Résumée : Dans cette communication, proposée en français, nous examinerons la métamorphose du lexique dans la langue parlée de l'est du Maroc; dans ce cas, celui des habitants des villes de Jerada et d'Ain Beni Mathar. Leur discours en passant d'une situation de communication ordinaire à une situation tendue, change complètement de registre. Les termes utilisés ne sont plus les mêmes. D'où le choix de l'expression métamorphose lexicale. Les termes et expressions chargés sémantiquement traduisent la colère et la détresse du locuteur. Dans ce langage de valeur dépréciatif, le discours est tissé en termes de métaphores et d'allégories. Il est également accompagné de gestes et ponctué de sons et d'intonations qui traduisent fidèlement le sentiment de rage qui envahit le locuteur.

Mots-clés : Vocabulaire, Lexique, Parler, Oriental marocain, Valeur dépréciative, Jerada, Ain Béni Mathar, Langue, Irritation, Mécontentement.

Abstract: «Vocabulary of irritation in the speech of eastern Morocco (Case of Jerada and Ain Béni Mathar)». In this communication, proposed in French, we will examine the metamorphosis of the lexicon in the oral language of the eastern region of Morocco; in this case that of the inhabitants of the cities of Jerada and Ain Beni Mathar. Their speech completely changes of register when it evolves from a situation of ordinary communication to a tense situation. The terms used are no longer the same; hence the choice of the expression "lexical metamorphosis". Semantically charged

\footnotetext{
Inspecteur principal de l'orientation scolaire. Direction Provinciale du Ministère de l'Éducation Nationale, de la Formation Professionnelle, de l'Enseignement Supérieur et la Recherche Scientifique - Oujda Angad. E-mail : medmalkim1966@gmail.com.

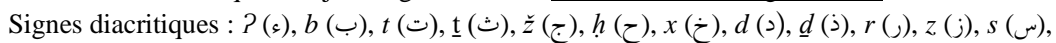

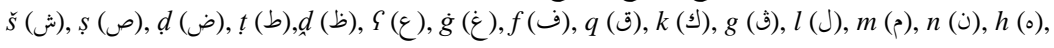
$w(\mathrm{~g}), y$ (s).
} 
terms and expressions convey the speaker's fury and distress. In this language of depreciative value, the discourse is woven in terms of metaphors and allegories. It is also accompanied by gestures, and punctuated by tones and intonations that faithfully translate the feeling of anger that invades the speaker.

Key words: Vocabulary, Lexicon, Speech, The East of Morocco, Depreciative value, Jerada, Ain Beni Mathar, Language, Irritation, Discontent.

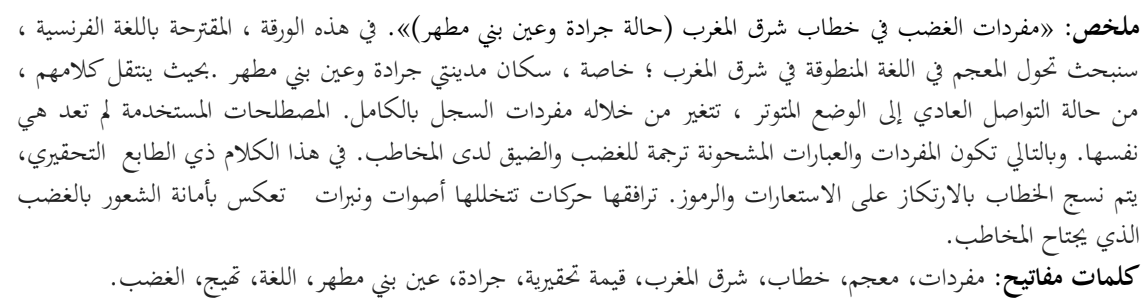

\section{Introduction}

Une question à caractère philosophique de type existentialiste s'applique au domaine de la recherche linguistique, à savoir, la relation qu'entretient l'homme avec la langue : qui dépend de l'autre ou qui agit sur l'autre ? Face à cette variété des langues et cette multiplicité des dialectes, on assiste à une floraison de termes et de nominations au sein d'une même langue ou dialecte. Et c'est dans le cadre de cette richesse et ces variétés linguistiques que nous entendons présenter cette communication abordant le Vocabulaire du mécontentement dans l'Oriental marocain (Cas de Jerada et Ain Béni Mathar).

Akouaou $(2001: 1)$ explique :

«Pour avoir accès au marché linguistique, en comprendre les fonctionnements, on peut tenter de l'approcher de deux manières : a) en cherchant à comprendre comment se positionnent les locuteurs par rapport aux différentes variétés i.e. comment d'une certaine manière, ils justifient à travers leurs jugements et expériences, les choix qui informent la compétition linguistique; b) en s'employant à définir les formes et la dynamique des contacts linguistiques».

Nous signalons que l'objet du présent travail s'écarte des deux démarches suscitées par Akouaou: nous nous limiterons à un stade statique basé sur l'observation et la description de quelques valeurs dépréciatives du parler de 
l'Oriental marocain à travers le lexique du mécontentement (cas de Jerada et Ain Béni Mathar).

Dans ce lexique, nous ferons apparaître le sentiment du mécontentement, de la haine ; de la dérision, de la satire et de l'insatisfaction.

Le corpus collecté se présente sous deux formes: des unités lexicales autonomes considérées hors du contexte; et des unités lexicales considérées dans un contexte tels que les proverbes, les expressions parémiques où le mécontentement ne se dégage qu'à travers le sous-entendu. Nous nous intéresserons, d'abord, dans le présent travail, à la première catégorie regroupant des unités lexicales autonomes considérées hors du contexte. Puis, nous entamerons l'étude des locutions regroupant des valeurs dépréciatives.

\section{Corpus}

1.1 Corpus des unités lexicales non contextualisées

ب/b/ būlām buâyra bəkāna mbəhlal ; būḥbaka.

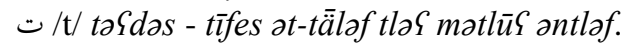

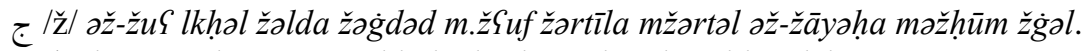

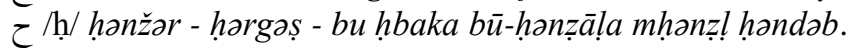

$\dot{\tau} / \mathrm{x} /$ xannus mxālat xarba.

د/d/ dalluz; dSaz; madৎūz; dahmūka; daSlag.

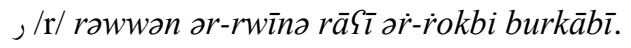

j/z/ m.zakram m.zəSzəS az-zalt mazlot zrag zläf.

س /S/ sawwak (humiliation); m-sakkar m-sahsah; m-santer m.sahwak; m.santah slugi mashug salgoṭ msalgat m.sarwal; as-s ārah maskūf; sandəf sūsag žll qəmməš mā yxāf mā yramməš bū sarbāna; msarwal sarrād.

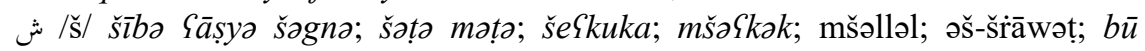
šrāwat.

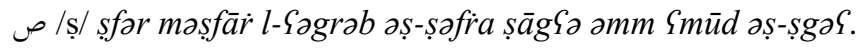



b/ț/ țlmeș mțarfaš.

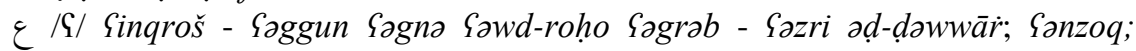
Sazwo.

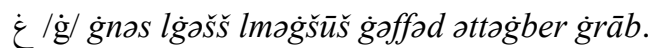

if/ farx; ganțoh; fanyǟn (a); farqaš.

ق /q/attaqber; mqazdar; l mdahwar; qazdira. 


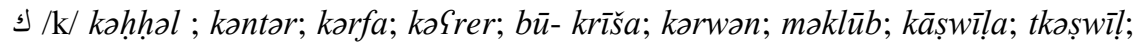
kəmmä̈ra ; kiḍār.

$\mathrm{J} / \mathrm{l} /$ mlaggat ; lafৎə; latmi; lti .

○/m/ maxxinna; mašlag.

ن/n/ m.nunax; nagৎa manhor; nqor; nakb.

$\mathrm{g} / \mathrm{w} /$ widah; mwaddah; wexxad.

ه /h/ hankāra; m.hịdar; hị̣or; haddāwi; həžžāla; mhadwan; həndrāza; harrma; harkë̈; mahrūso.

ث/g/ m- gatț̊ mgaršal mgazmot graৎ gaždar.

\subsection{Corpus des unités lexicales contextualisées}

- l-fās al-li yo-hfar Sli-ya yə-t-harros (la pioche qui cherche à me déraciner, qu'elle soit cassée).

- mā ya-dxal bin l-lham wa d- - far gi l-wsex (ne s'insère entre l'ongle et la chair que la saleté).

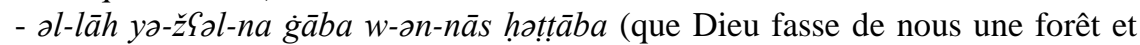
des autres, des bûcherons).= Nous ne serons plus gênés / provoqués par les gens qui nous médisent.

- glab l-gadra Sla femm-ha to-šbəh la-mm-ha (mets la marmite à l'envers, elle ressemblera à sa mère). $=$ Telle fille, telle mère.

- āš xașṣ-ek ā l-Garyān xașṣ-ni xātem ā mūlā-y (de quoi as-tu besoin ô nu, je voudrais une bague, monsieur).

- on-nsa ġi nsa waḥda Sla ž-žafna gālsa u w-weḥda bal-xulxāl nā̧sa (les femmes sont toutes pareilles; pourtant, l'une est pauvre, et l'autre mène riche). Il s'agit, ici, d'un cri face à l'inégalité sociale.

- t-saḥhar mSa d-drāri ta-ṣbạ fātar (si tu prends le repas du shur avec les gamins, ton jeûne n'aboutira pas). = si tu ne choisis pas tes coéquipiers avec précaution, tes projets seront voués à l'échec.

- al-lsān māḍi wa d-drā gāadi (la langue est aiguisée ; mais, le bras (travail) est néant).

- famm-ək laḥs-u kalb (que ta bouche soit léchée par un chien).

Dans ces locutions nous essayerons de montrer la valeur dépréciative en dégageant les procédés linguistiques et métaphoriques utilisés pour atteindre cette fin. 


\section{Analyse}

\subsection{Analyse du corpus des unités lexicales contextualisées}

Le présent sujet sera abordé, suivant deux niveaux : des unités lexicales autonomes et des unités lexicales contextualisées. Étant donné que « le lexique d'une langue ne se compose pas seulement de mots simples, mais aussi d'expressions et de mots composés. Autrement dit, il comprend aussi bien des unités monolexicales que des unités polylexicales » (Saad Ali 2016: 103). Sur le plan discursif, le recours aux expressions figées, constitue une stratégie, à la fois, défensive et offensive cherchant à embarrasser l'adversaire. Dans un premier temps, nous examinerons le corpus des unités lexicales contextualisées ; puis, étudierons le corpus des unités lexicales libres.

\subsubsection{Prédominance des formes globalisantes}

Il s'agit de l'emploi des déictiques à la troisième personne, des formes impersonnelles, formes passives, où on ne pointe pas du doigt la personne visée par le discours : une personne $\mathrm{X}$, monsieur tout le monde, comme dans :

- l-fās al-li ye-ḩfor Sliya yo-t-harras (la pioche qui cherche à me déraciner, qu'elle soit cassée). = Que Dieu se venge de celui qui cherche à me causer des ennuis.

- al-lsān māḍi wo d-drā̧ gādi (la langue est aiguisée ; mais, le bras (= travail) est nul). = On parle trop, mais, on ne réalise rien.

L'emploi de ces formes généralisantes, viserait, sans doute, à intimider l'interlocuteur et à l'embarrasser, car il ne peut pas riposter à cette «attaque » verbale quoiqu'il soit convaincu que c'est lui «la pioche, le perturbateur, le méchant...».

Derrière la troisième personne, se déguise un interlocuteur, un « tu/ vous » comme dans : - mā ya-dxal bin l-lham wa d-dfar gi l-wsax (Ne s'insère entre l'ongle et la chair que la saleté). = Vous, qui vous mêlez des affaires des autres, vous êtes une saleté. Donc, ici, on assiste à une permutabilité permanente entre les déictiques de la troisième et de la deuxième personne. 


\subsubsection{La communication fictive}

L'emploi des métaphores, accentue l'expressivité de ces formes figées. l-fas (ou pioche) réfère à la personne ennuyeuse, méchante ; al-lsān mādi (= la langue aiguisée), personne bavarde, qui critique les gens; l-gadra (= la marmite) dans glab l-gadra Sla famm-ha tə-šbəh la-mm-ha (mets la marmite à l'envers, elle ressemblera à sa mère). $=$ Telle fille, telle mère ; mais, ici, on met l'accent sur le sens péjoratif de l'expression en laissant entendre que l'interlocuteur à les mêmes défauts que sa mère (voire ses parents, membres de sa tribu...).

\subsubsection{Une confrontation face à face}

A travers les déictiques renvoyant à la deuxième personne, s'établit une communication directe qui restreint le champ de l'implicite. On ne se réserve plus dans son discours. La personne concernée est désignée du doigt. C'est le cas de ces trois exemples :


gamins, ton jeûne n'aboutira pas). $=$ si tu ne choisis pas tes coéquipiers avec précaution, tes projets seront voués à l'échec.

- āš xașş-ək a $\bar{l}$ l- Gəryān xașs-ni xātam ā mūlāa-y (de quoi as-tu besoin ô nu, - je voudrais une bague, monsieur). $=$ Toi qui t'intéresses au le superflu, saches qu'il y a des choses plus importantes.

- famm-ək lahs-u kəlb (que ta bouche soit léchée par un chien). Cette locution résiste à la traduction comme «beaucoup d'expressions figées [qui] sont chargées d'implicites sociaux et culturels » (Saad Ali $2016: 104)$; donc, il est difficile de traduire fidèlement sa signification pour les non-natifs et qui ne sont pas imprégnés de la culture locale de la région. Partageant la même idée, Tamba (2011 : 116) souligne que l'opacité sémantique d'une expression figée est ainsi bien rattachée à la non-compositionnalité de son sens, qui recèle souvent un aspect métaphorique. Pour rapprocher le sens, nous dirons simplement qu'il s'agit d'une réprimande destinée à quelqu'un qui vous annonce une mauvaise nouvelle ou prédiction, de peur qu'elle se réalise.

A travers ces énoncés, la valeur dépréciative constitue une manière de s'imposer à travers le discours. On y a recours pour tonifier ses arguments, réfuter des thèses ou, tout simplement, manifester sa colère. Soulignons que, parfois, les zones entre l'ironie et la colère sont des zones amorphes concourant au même sens où une même expression se prête à une double lecture : compositionnelle et non compositionnelle (figée) (Palma 2007 : 23). 


\subsection{Analyse du corpus des unités lexicales non contextualisées}

Comme nous l'avons déjà mentionné ; le trait commun qui unit les termes de ce corpus c'est leur appartenance au champ de la colère et de l'insatisfaction à propos d'une situation, d'un être; d'un objet ou de soi-même. Cet état d'âme mène l'interlocuteur, en quête d'un refoulement, à se venger, en premier lieu, de la langue en modelant ses mots, en les fracassant, en les dénaturant pour leur donner, à sa guise d'autres formes et leur procurer d'autres sens. C'est l'insurrection contre l'ordre établi. Nous assistons, alors, à une foire où l'insurgé, lors de sa révolte, use de l'arme de la métaphore et de la caricature pour ridiculiser, minimiser, intimider son/ses interlocuteur(s)

Aussi, peut-on assister à une stratification des champs sémantiques que nous schématiserons de la manière suivante :

\subsubsection{Un lexique de genre}

Dans notre corpus, nous avons pu constater des termes qui sont propres au genre féminin : bāayra bəkāna. Par contre, d'autres ne s'emploient qu'avec le masculin : $\partial \dot{r}-\dot{r} o k b i-b u r k a \bar{b} \bar{i}$.

Cependant, un certain nombre de termes, qui devraient s'employer, par règles de bienséance et de convenance, à l'un des deux sexes, sont affectés, par ironie et sadisme à l'autre: Gəzri ad-dəwwwār (gaillard du quartier) terme masculin pour désigner une fille dans le but de la ridiculiser. Les termes hargaṣ ; səwwak; $̧ ə k k \partial \dot{r}$ relatifs au maquillage, sont attribués à des personnes de sexe masculin pour les ridiculiser.

\subsubsection{Un lexique des vêtements}

Pour critiquer la posture ou se moquer d'une personne, on met en scène les habits qu'elle porte : m-gazmat m-hadwan haddāwi hankāra harkäs m-qazzab.

\subsubsection{Un lexique d'animaux}

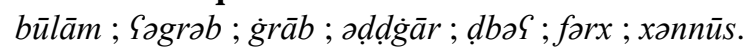

\subsubsection{Un lexique de défaillance morale}

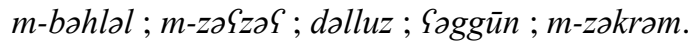


2.2.5. Un lexique de défaillance physique

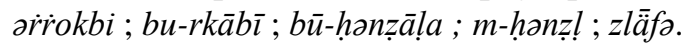

2.2.6. Un lexique du rang social

az-zalt ; mazlut ; m-gattol; m-bordi.

2.2.7. Un lexique de vices

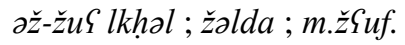

2.2.8. Un lexique de couleurs

ż̀g ; sfár ; maşfār.

2.2.9. Un lexique de proximité

tälaf; tlas; matlu $\varsigma$; antlaf.

2.2.10. Un lexique de table

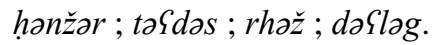

\subsection{Quelques notes sur la morphologie}

En examinant la forme des termes de notre corpus, nous avons constaté qu'ils se répartissent comme suit :

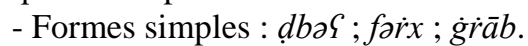

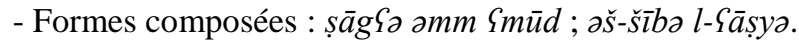

- Formes verbales : ġnas; daఢlag; hanžar.

- Formes adjectivales : sfar; zrag.

- Formes nominales : $\underset{b}{b \partial}\} ;$ farx ; $\dot{g} r a \bar{b}$.

- Formes participe : mə-hrūsa; m-gatț̊ ; m-garšal; m-gazmət.

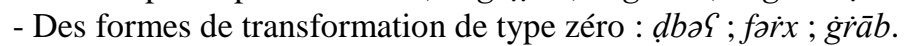

- Des formes de transformation partielle : loț/mi pour xud/mi (couteau); $\partial s$ sarrād pour barrād (théière).

- Des formes de transformation totale : ḍlāḥ (l-ma /eau) ; lṭi (jdi/chevreau).

\subsection{La valeur dépréciative du lexique}

Pour mettre à vue la valeur dépréciative de ce jargon, il serait préférable d'y procéder par le concours de plusieurs approches: communicative, discursive, sémantique, pragmatique et voire sémiotique. D'où, faudrait-il 
considérer ce lexique dans son contexte. Et quoique nous l'ayons abordé, dans cette partie, en tant qu'unités lexicales autonomes, nous pourrons, quand même, tenter de dégager quelques-unes de ces valeurs.

Ainsi, pour exprimer une critique à l'égard d'un être, d'un objet ou d'une situation, on utilise une batterie de termes ou d'expressions, pour mettre en scène soit le sentiment de colère, d'humiliation, de menace, d'irritation, ou d'intimidation.

A titre d'exemple, nous présentons, ici sous forme d'anecdote, le discours tenu par un paysan qui se voit dans l'obligation de recevoir pour le dîner des invités non désirés. Sous la tension de l'irritation, il s'adressa à son fils :

- gūl l- mūmit-k twažžad as-sarrād w žlb al-lațmi bāš nanḩar al-lți. Si le père n'avait pas été soit disant irrité et avait su garder son calme, il aurait pu dire : - gūl l-a mmə-k twəžžวd al-barrād w žīb al-xūdmi bāš nədbəḥ əž-ždi.

La traduction de ce discours serait : Dis à ta mère de préparer la théière et apporte le couteau pour égorger le chevreau.

\begin{tabular}{|c|c|c|}
\hline Termes à valeur dépréciative & Termes courants & Équivalent \\
\hline$m \bar{u} m i t-k$ & ә $m m \partial-k$ & ta mère \\
\hline as-sarrād & al-barrād & la théière \\
\hline al-laṭmi & ol-xūdmi & le couteau \\
\hline nənḩor & nadboḥ & égorger \\
\hline al-ltti & $\partial \check{z}-z \check{z} d i$ & le chevreau \\
\hline
\end{tabular}

\section{Glossaire}

\section{ب/b/}

bāyra بائرة : n.f. sing. ; pl. -āt; grande fille ayant dépassé l’âge du mariage.

bəkāna بكانة : n.f. sing. ; pl. -āt; fainéante et non expérimentée.

mbahlal مبهـ: n.m. sing. ;f. - $a$; m.pl. -īn ; f. pl. -āt; débile ; niais.

būlām بولام: n.m. sing. ; sans f.; sans pl. individu indigne de respect (un lézard).

büḥbaka بوحبكة : n.m. sing. ; sans f.; sans pl. personne peu intelligente, qui se laisse facilement, duper par les autres.

\section{$ت / \mathbf{t} /$}

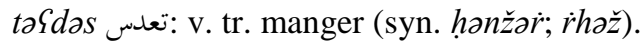

tīfas تيفس :ت intr. devenir aveugle. 
tǟllaf تالف: adj.m.s; f. -a ; m.pl. -inn; f.pl. -āt ; batârd, dont on ignore l'origine.

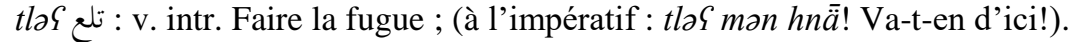
antlaff انتلف / انلف : v. intr. disparaitre (à l'impératif : tlaf mən hnä! Va-t-en d'ici !).

ج|冖̌z $\mid$

až-žuৎ lkhal lلكوع الكحل : (nom composé) : personne très avare et avide (syn. žalda).

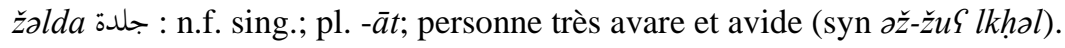

žəg $\dot{g} d \partial d$ : : ve intr. Entretenir un discours long répetétif et agaçant.

$m z ̌ \bar{u}\{f$ مجوعف : adj.m. sing. ;f. - $a$; m.pl. -īn ; f.pl. -āt; personne très avide.

žrtīila جرتيل : n.f. sing. ; pl. -āt; individu sale.

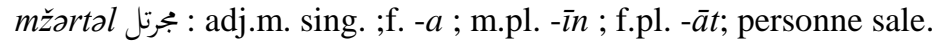

žāyəh جايح : adj.m. sing. ;f. - $a$; m.pl. -ìn; f. pl. -ät; paresseux.

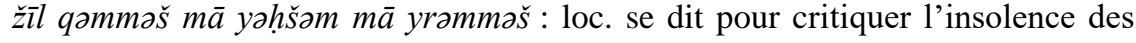
jeunes.

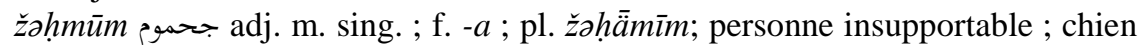
errant.

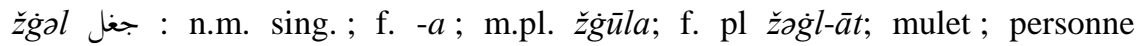
grossière ; mal élevé.

г/h/

ḥlwəš حلوش : v. tr (iron.) manger voracement; amasser de l'argent avec avidité (Sabia et Al. $2004: 115$ ).

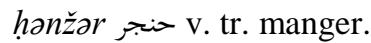

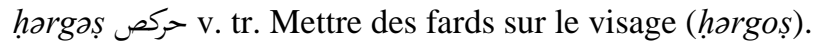

bu hbaka بوحباكمة n.m. sing. ; sans f.; sans pl.; individu inexpérimenté ; faible.

$b \bar{u}-h \partial n z \bar{a} l a l a$ بوحنزالة n.m. sing. ; sans f.; sans pl.; individu ayant un gros ventre.

həndəb حندب v. intr. Se lamenter; se griffer le visage par tristesse (syn. gəždər).

$\dot{\tau}^{/ \mathbf{x} /}$

xənnūs خنوس n.m. sing. ; sans f.; pl. xnānīs. Petit enfant qui ne cesse de crier. mxālot خ adj. mas. ; f. - $a$; pl. m. -inn ; pl.f -ät; hanté par des esprits.

xərba خربة n.f. sing. ; pl. xrab ; vieille demeure qui est sur le point de s'écrouler (syn. nqor). 
$2 / \mathbf{d} /$

dalluz adj.m. sing. ; f. $-a$; m. pl. dlälaz; f. pl. -āt; personne naïve (syn. Soggūn).

$d \varphi_{\partial z}$ دع.m. sing. ; sans f; sans pl. 1- nourriture empoisonnée ; 2- corruption madGiuzz مدعوز: adj. m. sing.; f. $-a$; m. pl. -ìn.; f. pl. -āt; 1- personne empoisonnée 2- personne corrompue.

dahmūka دهوكة : n. f. sing.; f. pl. -āt; 1- tête grossière 2- personne insupportable.(syn. kəmmāro).

dəSlog دعلك : v. tr. boire.

,$/ \mathbf{r} /$

rawwan وون : v. tr. ; faire un travail incorrect.

or-rwiñn روينة : n. f. sing. travail mal fait ou son résultat.

rā

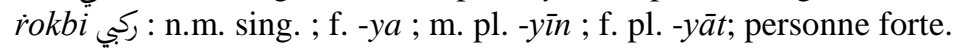

bu-rkā̄o بوركابي : n. m. sing. ; sans f.; sans pl. individu à jambes longues et musclées.

$j / \mathbf{z} /$

m-zakram مزكرم : adj. m. sing. ; f. - $a$; m. pl. -īn ; f. pl. -āt; Individu replié sur soi.

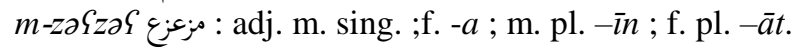

$z$ zlt

mazlot مزلوط : n. m. sing. ; f. - $-a$; m. pl. -īn; f. pl. -āt; personne démunie.(syn. $m g \partial t \operatorname{tt}()$.

$\operatorname{zrag} \mathcal{F}^{2}$;: adj. m. sing. ;f. $-a$; m. pl. -īn ; f. pl. -āt; nullard.

$z l a ̈$ fa زلافة : n. f. sing. f. pl. -āt; gueule; figure (syn. kəmäära).

س/s/

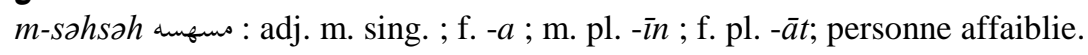

$m$-santer مسنr : adj. m. sing.; f. $-a$; m. pl. $-\bar{l} n$; f. pl. -àt; etre sans le sous, difficulte materielles.

$m$-səntวh مسنته : adj. m. sing. ; f. $-a$; m. pl. -īn; f. pl. -āt; personne passive et qui manque la vivacité.

slugi سلوفي : n.m. sing. ; f. -ya ; f. pl. -āt; m. pl. släg chien de chasse.

mashugg مسحوث : n. m. sing. ; f. -a ;; f. pl. -āt; m. pl. msaḥigg. 
m.sarwal مسرول: adj. m. sing. ; f. - $a$; m. pl. ìn ; f. pl. -āt; personne mal vêtue. sārah سارح : n. m. sing. ; f. $-a$; m. pl. -īn ; f. pl. -āt; berger.

maskūff مسكوف: adj. m. sing. ; f. - $a$; m. pl. -īn ; f. pl. -ät; personne courbée . sūsag سوسث : v. intr. Errer d'une lieu a un autre.

bu sarbāna بوسربانة : n.m. sing. ; sans f.; individu mal habille.

štotbo شطبة : n. f sing. ; f. pl. -āt; une femme ou fille difficile a dominer, hommasse.

šṭība شطيبة : n. f sing. ; f. pl. -āt; diminutif de šoṭbo.

sərrād سراد : n. m sing. ; pl. srārad, théière.

ش/ $/$

Šlba Sāṣya ش. n. composé une personne avancée dans l'âge et qui dévie du droit chemin.

šagnว شڤنة : adj.f. sing. ; f. pl. -āt; individu peureux qui ne peut pas se défendre (syn. būḥbaka).

Šaț moț شاطة : n. composé, femme ou fille imbattable (syn. șāgৎa amm ९mūd; Sawd-roho).

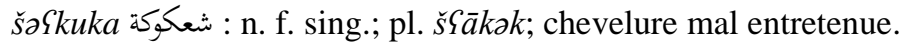

mšallal مشلل: adj. m. sing. ; f. - $a$; m. pl. -in ; f. pl. -āt; nullard (syn. zrag).

šgfa شخَّة : n. f. sing .; pl. -ât, une vieille personne.

ص/ș/

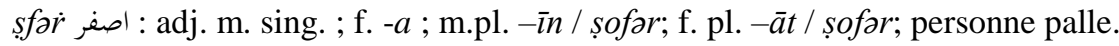
mașfāri مصفار : n. m. sing. ; f. - $a$; m. pl. -īn ; f. pl. -āt; personne pâle.

l-Gagrab aș-şafra العفرب الصفرة: n. f composé, personne très sévère (scorpion). șāg (syn. šato mota ; Gawd-roho).

șalgoṭ سلثوط : n. m. sing. ; f. -ya ; f. pl. -āt; m. pl. șlāgət, personne mal élevée.

ض/ḍ/

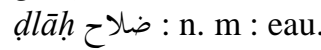

dərnoh ضرنوح : n. m. sing. ; f. - $a$; f. pl. -āt; m.pl. ḑrānīḥ; enfant très agaçant.

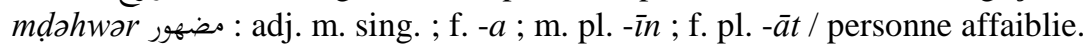

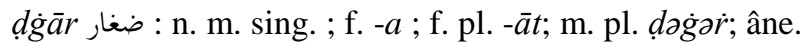




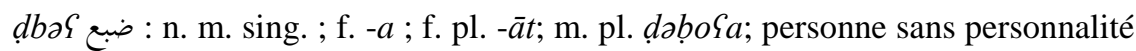

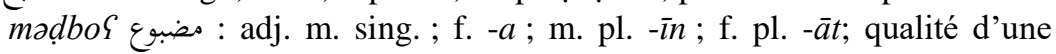
personne faible.

b/ț/

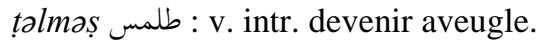

mtalmaș مطلمس : adj. m. sing. ; f. - - ; m. ml. -īn ; f. pl. -āt; aveugle.

$m$ marfašs مطرفش adj. m. sing. ; f. - $a$; m. pl. - -in ; f. pl. - $\bar{a} t$; stupide.

$\varepsilon / \mathbf{S} /$

Ginqruš عين قروش : n. f. composé sing. ; sans m; sans pl.; une femme très sévère.



Sagna عثنة : n. f. sing.; f. pl. -ät; (dimunitif : Sginna).

Səwd-roho عود روحو: n. m. sing.; sans f.; sans pl. , une femme très sévère ;

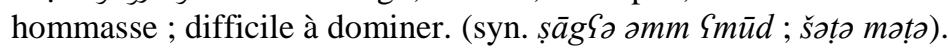

\agrab عثرب: n. f. sing. ; sans m. pl. §gä̈reb; une femme très sévère.

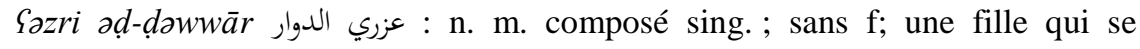
comporte comme un garçon (syn. züfri).

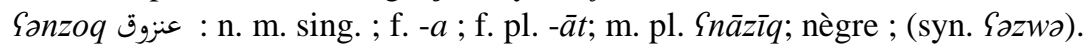

Səzwə عزوة : n. f. sing.; sans m; pl. Səzwāt ; nègre; (syn. Sənzoq) (s'emploie indifféremment pour les deux sexes).

$\dot{\varepsilon} / \dot{\mathbf{g}} /$

$\dot{g} n \partial s$ غنس : v.tr. (pour une femme) mettre une pièce d'étoffe pour se couvrir la tête et le visage pour éviter le regard des étrangers; (pour l'homme) se dit pour le ridiculiser.

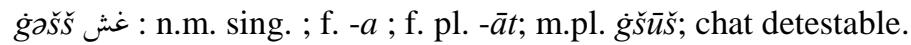

attag்ber اتغبر : v. int. dormir; (syn. attəqber); Quand on est fâché contre quelqu'un, au lieu de lui dire «sìr tanSes va dormir!» on lui dit «sìr attəg்bar».

$\dot{g} r a \bar{a} b$ اغراب : n. m. sing.; sans f; pl. əg்raḅba ; se dit par superstition d'une personne indésirable (syn. $m \bar{u} k a$ ) (s'emploie différemment pou les deux sexes). 
ف/f /

fərx فرخ : n.m. sing. ; f. - $a$; f. pl. -ät; m. pl. frūuxa / mffärex; bâtard ; mal élevé. gantoh fanyän (a) فنيان : adj. m. sing. ; f. -a ; m. pl. -inn ; f. pl. -āt; fainéant.


quelqu'un qui s'asseoie en allongeant ses pieds, on dit : '《žməऽ fräqšek » = Assis-toi correctement.

\section{ق /q/}

attaqber اتقبر : v. int. dormir; (syn. attaǵber); Quand on est fầché contre quelqu'un,

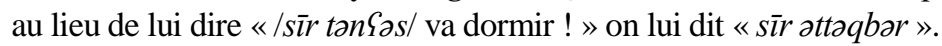

qazdira قزديرة: n.f. sing. ; sans m; pl. qzāder / qazdīrät ; tout engin vieux et usé.

mqazdar مقزدرة adj. m. sing. ; f. - -a; m. pl. -īn; f. pl. -āt ; impoli ; qui lance des mots grossiers devant tout le monde.

s / k/

kahhal كحل : v. tr. Mettre du khol dans les yeux; le mot utilisé par/pour les hommes pour exprimer un défi " ila nta taffartah äji kəhḥ̆al-li » Trad. Je te défi de réussir; et si tu réussis, je mettrai du khol dans mes yeux telle une femme. (syn. səwwak; §əkkər; hənni; hargas).

tkantar تكنتر: v. int. se dit lorsqu'on se trouve dans l'impasse devant un problème; syn. tkarwan.

karfa كرفة : n. f. collectif ; sans pl. résidu ; groupe de personnes sans mérite.

kəSrer كعرة : v. int. Revenir sur ses paroles ou ses principes.

$b \bar{u}-k r i \bar{s} a$ a بو كريشة : n. m. composé sing. ; f. / üm-krī̌sa; sans pl.; qui a un gros ventre.

tkarwan كرون : v. int. se dit lorsqu'on se trouve dans l'impasse devant un problème; (syn. tkantar).

məklūu مكلوب: adj. m. sing. ; f. - -a ; m. pl. -īn ; f. pl. -āt; enragé.

kāṣswịla كاصويلة : adj. f. sing. ; f. pl. -āt; fainéant (e) (s'emploie indifféremment pour les deux sexes. (syn. fanyān).

tkașwīl lتكصويل : n. m. sing. sans f. sans pl. action de fainéanter.

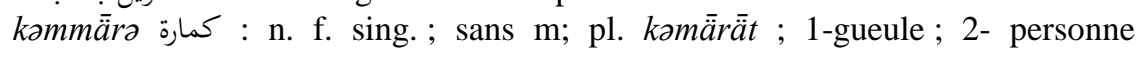
insupportable (syn. dəhmūka).

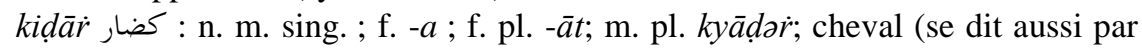
mépris à une personne). 
$\mathrm{J} / \mathrm{l} /$

mlaggat ملثط : adj. m. sing. ; f. - $a$; m. pl. -īn ; f. pl. -āt; bâtard (syn. farxx).

lafৎa لفعة : n. f. sing. ; sans m; pl. -āt; une femme très sévère (syn. Ginqroš Gagrab).

lațmi لطمي : n. m. sing. ; sans f.; pl. lțāma; couteau.

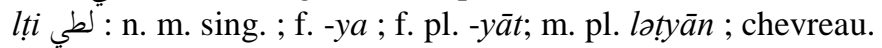

م/m/

moxxīna مخينة : n. s. sing. ; sans m ; f. pl. -āt; une femme sale.

məšl lag مشلث : n. m. sing. ; sans f.; pl. məšălag, une femme très sévère ; hommasse ; difficile à dominer. (syn. 〔awd-roho @azri aḍ-dawwāri).

$m \bar{u} k a$ موكة : n. f. sing. ; sans m; pl. mūkāt ; se dit par superstition d'une personne indésirable (syn. $\dot{g} r a \bar{b} b$ ) (s'emploie différemment pou les deux sexes).

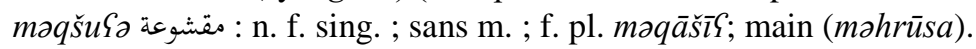

ن /n/

mnunəx منونخ : adj. m. sing. ; f. - $a$; m. pl. -in ; f. pl. -āt; fainéant; personne affaiblie ; (syn. mọdəhwor).

nəgโว نثعة : n. f. sing. ; sans m.; pl. nagৎăt; individu peureux qui ne peut pas se défendre; personne irresponsable ; stupide. (syn. šagnə).

nqor نقور : n. m. sing. ; sans f. pl. nqora; vieille demeure qui est sur le point de s'écrouler (syn. xərbə).

nəkb نكب: n. m. sing. ; sans f.; sans pl ; grains de blé qu'on jette aux poules ; la nourriture.

g/w/

widdoh ويدح: ve int. Se lamenter ; pleurer sa mal-chance.

twīdīh تويديح : in.m ; sans f. sans pl. ; lamentation.

mwaddahh مودج : adj. m. sing. ;f. - $a$; m. pl. -īn ; f. pl. -āt; très sale (syn. mgaršal).

twexxad توخض : v. (forme passive) subir une grande perte ; faire faillite.

$\rightarrow / \mathbf{h} /$

hənkāra هنكارة : n. f. sing. ; sans m; pl. hənākīr / hənkārāt ; vielles chaussures (syn. harkās).

mhīẹdr مهيض : adj. m. sing. ; f. - $a$; m. pl. -inn ; f. pl. -ät; très maigre.

hị̂dor هيضور: n. coll. Troupeau très maigre. 
həžžāla هجالة : n. f. sing. ; sans m; f. pl.; -āt 1-veuve 2- une fille dont la virginité est mise en cause.

haddāwi هداوي: n. m. sing. ; f. -ya ; f. pl. -yāt; m. pl. haddāwa ; personne mal vêtue.

həndrāza هندرازة : n. f.. ; sans m; pl. -āt; objet très lourd.

hərrm هرمة : n. f. sing. ; (désignant les deux sexes) ; sans pl. ; personne atteinte de boulémie ; mange-tout.

hərkās هركاس : n.m. sing. ; sans f.; pl. hräkīs; vielles chaussures (syn. hənkāra).

məhrūsa مهروسة: n. f. sing. ; sans m.; pl. mhä̈rīs; main (syn. məašū

†/g/

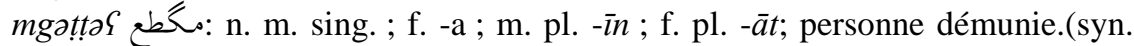
mazlot).

mgaršal مثـرشل : adj. m. sing. ; f. - $a$; m. pl. -īn; f. pl. -āt; très sale (syn. mwəddə⿳亠).

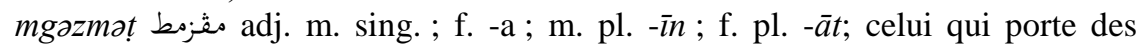
habits plus petits que sa taille (syn. mqazzab).

grə؟

gaždar فُشجدر: intr. Se lamenter; se griffer le visage par tristesse (syn. handab).

\section{Conclusion}

L'expérience que nous venons de vivre à travers la réalisation de ce modeste travail nous a permis de tirer quelques conclusions. Nous les résumerons dans les points suivants :

- Notre patrimoine oral constitue un champ fertile pour les recherches qui s'inscrivent dans le domaine de la dialectologie.

- Il est temps de réfléchir à réunir les efforts pour réaliser un atlas du parler de l'Oriental marocain pour découvrir ses spécificités, sa richesse et les variétés linguistiques de cette région.

- L'opérationnalisation du dernier point serait par la constitution des groupes de recherches qui mèneraient des enquêtes dans toutes les tribus de l'Oriental marocain.

- Les résultats auxquelles auraient pu aboutir ces enquêtes devraient faire l'objet d'un colloque dans le but de finaliser et affiner les travaux et donner le coup d'envoi à la réalisation du dit Atlas linguistique des parlers de l'Oriental marocain. 
- Penser à faire sortir au monde un Atlas linguistique des parlers de l'Oriental marocain n'est plus une utopie ; il suffit de prendre connaissance des projets qui se réalisent dans le même sens au niveau national: nous citerons à titre d'exemple le projet "Parlers et Société Jbala" où sont impliqués des organismes nationaux et internationaux.

\section{Appendice : entretiens, 12 informateurs}

\begin{tabular}{|l|l|}
\hline Informateur $\mathrm{n}^{\circ} 1$ & Informateur $\mathrm{n}^{\circ} 2$ \\
Nom : SMAILI Messaouda & Nom : SMAILI Brahim \\
Age : environ 80 & Age $: 75$ \\
Natif de : Ain Béni Mathar & Natif de : Jerada \\
Profession : Sans & Profession : Professeur \\
Lieu de résidence : Ain Béni Mathar & Lieu de résidence :Jerada \\
Situation familiale : veuve & Situation familiale : marié \\
Niveau d'instruction :sans & Niveau d'instruction : niveau bac \\
Tribu :Chorfa Ouled Moulay Abdelmalek & Tribu : Chorfa Ouled moulay Smail \\
\hline
\end{tabular}

\begin{tabular}{|l|l|}
\hline Informateur $\mathrm{n}^{\circ} 3$ & Informateur $\mathrm{n}^{\circ} 4$ \\
Nom $: Z A R H O U N I$ Fatna & Nom $:$ HASSOUNI Ali \\
Age $: 65$ & Age $: 35$ \\
Profession : Sans & Profession : menuisier \\
Lieu de résidence : Jerada & Lieu de résidence $:$ Jerada \\
Situation familiale $:$ veuve & Situation familiale : marié \\
Niveau d'instruction : Primaire & Niveau d'instruction : collège \\
Tribu : Zkara & Tribu : Ouled Sidi Ali \\
\hline
\end{tabular}

\begin{tabular}{|l|l|}
\hline Informateur $\mathrm{n}^{\circ}$ 5 & Informateur $\mathrm{n}^{\circ} 6$ \\
Nom : LFERH Chama & Nom : LFERH Slimane \\
Age : 48 & Age : 35 \\
Profession : Sans & Profession : Commerçant \\
Lieu de résidence : Ain Béni Mathar & Lieu de résidence : Ain Béni Mathar \\
Situation familiale : mariée & Situation familiale : marié \\
\hline
\end{tabular}


Niveau d'instruction : primaire

Tribu : Bni Guil

Informateur $\mathrm{n}^{\circ} 7$

Nom : FELGHOUMI Hassan

Age : 26

Profession : fonctionnaire

Lieu de résidence : Jerada

Situation familiale : Célibataire

Niveau d'instruction : $\mathrm{Bac}+2$

Tribu : Beni Amer
Niveau d'instruction : secondaire Tribu : Bni Guil

Informateur $\mathrm{n}^{\circ} 8$
Nom : ELASRI Najat
Age : 22
Profession : Etudiante
Lieu de résidence : Ain Béni Mathar
Situation familiale : célibataire
Niveau d'instruction : bac
Tribu : Béni Mathar

\begin{tabular}{|l|l}
\hline Informateur $\mathrm{n}^{\circ} 9$ & Informateur $\mathrm{n}^{\circ} 10$
\end{tabular}

Nom : BAHHOUSSI Bouamama Nom : OUCHEN Mohamed

Age : 49

Profession : Boucher

Lieu de résidence : Ain Béni Mathar

Situation familiale : marié

Niveau d'instruction : secondaire

Tribu : Oulad Sid Chikh

Age : 26

Profession : commerçant

Lieu de résidence : Jerada

Situation familiale : marié

Niveau d'instruction : secondaire

Tribu : Ghouat

\begin{tabular}{|l|l|}
\hline Informateur $\mathrm{n}^{\circ} 11$ & Informateur $\mathrm{n}^{\circ} 12$ \\
Nom $:$ RAHMANI Zineb & Nom : ARABI Issam \\
Age $: 33$ & Age $: 18$ \\
Profession : couturière & Profession : étudiant \\
Lieu de résidence $:$ Jerada & Lieu de résidence $:$ Ain Béni Mathar \\
Situation familiale $:$ divorcée & Situation familiale $:$ célibataire \\
Niveau d'instruction $:$ collège & Niveau d'instruction : bac \\
Tribu : Tsoul & Tribu : Zkara \\
\hline
\end{tabular}

$* * *$ 


\section{BIBLIOGRAPHIE}

AKOUAOU, Abdelali. 2001. "Compétition et hiérarchie linguistiques au Maroc ». Revue de la Faculté des Lettres et des Sciences Humaines Dhar El Mahrez-Fès 12, pp. 1-13.

AKOUAOU, Abdelali. 1997. "Les variétés linguistiques au Maroc. Statuts, usages et fonctions». Dans: Voisinage, mélange en hommage à la mémoire de K. Cadi. Miloud Taïfi (éd.). Fès, pp. 67-88.

BOUKOUS, Ahmed. 1995. Société, langues et cultures au Maroc, enjeux symboliques. Rabat. Publication de la Faculté des Lettres, Série Essais et études.

LAROUSSI, Foued. 1997. Plurilinguisme et identités au Maghreb. Rouen, Publications de l'Université de Rouen et du Havre.

PALMA, Silvia. 2007. Les éléments figés de la langue, étude comparative français-espagnol. Paris, L'Harmattan.

SABIA, Abdelali \& al. 2004. Dictionnaire Arabe-Français de Langue et de Culture Marocaine (Maroc Oriental). Oujda. Publication de FLSH.

TAMBA, Irène. 2011. «Sens figé : idiomes et proverbes ». Dans : Le figement linguistique : La parole entravée. Anscombre, Jean-Claude \& Mejri, Salah (éds.). Paris, Éditions Champion, pp. 109-126.

SAAD ALI, Mohamed. 2016. «La traduction des expressions figées : langue et culture ». Traduire 235, pp. 103-123.

<https://journals.openedition.org/traduire/865> [22 août 2019]. 